\title{
Author Correction: STING and IRF3 in stromal fibroblasts enable sensing of genomic stress in cancer cells to undermine oncolytic viral therapy
}

Esther N. Arwert, Emma L. Milford, Antonio Rullan (D), Stefanie Derzsi, Steven Hooper, Takuya Kato, David Mansfield, Alan Melcher, Kevin J. Harrington and Erik Sahai (iD

Correction to Nature Cell Biology https://doi.org/10.1038/s41556-020-0527-7, published online 1 June 2020

In the version of this article originally published, there were errors in Extended Data Fig. 1 and its legend. In Extended Data Fig. 1a, the labels for the experimental scheme were swapped. 'Indirect Coculture (No Touch)' should be under the left image and 'Direct Coculture (Touch)' should be under the right image. Additionally, in the legend, panel ' $\mathrm{d}$ ' was cited twice. Those panels in the legend should read "D) qRT-PCR of ISGs MX2 and OAS2 of the co-cultures of the non-transformed epithelial HaCat cell line with VCAF2b compared with A431/VCAF2b co-culture. Each dot is a biological replicate $(n=3$ independent experiments, except for OAS2 A431 control sample $n=$ 2). E) qRT-PCR of ISGs MX2 and OAS2 of co-cultures of A431 with HUVEC compared with A431/VCAF2b co-culture. Each dot is a biological replicate ( $n=3$ independent experiments). F) qRT-PCR of ISGs MX2 and OAS2 of co-cultures of A431 with PBMC-derived macrophages from two different donors, compared with A431/VCAF2b co-culture. Each dot is a biological replicate $(n=4$ independent experiments for A431-Macrophages and $n=3$ for A431-VCAF2b)." The errors have been corrected in the PDF and HTML versions of the paper.

Published online: 18 June 2020

https://doi.org/10.1038/s41556-020-0544-6

(๑) The Author(s), under exclusive licence to Springer Nature Limited 2020

\section{Publisher Correction: ROCK-mediated selective activation of PERK signalling causes fibroblast reprogramming and tumour progression through a CRELD2-dependent mechanism}

Sarah Theresa Boyle (iD, Valentina Poltavets, Jasreen Kular, Natasha Theresa Pyne, Jarrod John Sandow (D), Alexander Charles Lewis, Kendelle Joan Murphy, Natasha Kolesnikoff, Paul Andre Bartholomew Moretti, Melinda Nay Tea, Vinay Tergaonkar, Paul Timpson, Stuart Maxwell Pitson, Andrew lan Webb, Robert John Whitfield D, Angel Francisco Lopez, Marina Kochetkova (D) and Michael Susithiran Samuel $\mathbb{D}$

Correction to Nature Cell Biology https://doi.org/10.1038/s41556-020-0523-y, published online 25 May 2020

In the PDF version of this article originally published, a text label was omitted from Fig. 2g. The heading for the images in the right-hand column of Fig. $2 \mathrm{~g}$ should be "R-PyMT $+4 \mathrm{HT}$ ". The error has been corrected in the PDF version of the paper.

Published online: 4 June 2020

https://doi.org/10.1038/s41556-020-0539-3

(c) The Author(s), under exclusive licence to Springer Nature Limited 2020 